$01,05,11$

\title{
Влияние гидрирования на структуру, магнитные и магнитокалорические свойства сплавов Tb-Dy-Co со структурой фаз Лавеса
}

\author{
(С) И.С. Терёшина ${ }^{1}$, Т.П. Каминская ${ }^{1}$, В.Б. Чжан ${ }^{1}$, Ю.А. Овченкова ${ }^{1}$, А.С. Трушева ${ }^{1}$, А.А. Вирюс ${ }^{2}$ \\ ${ }^{1}$ Московский государственный университет им. М.В. Ломоносова, \\ Москва, Россия \\ ${ }^{2}$ Институт экспериментальной минералогии РАН, \\ Черноголовка, Россия \\ E-mail: irina_tereshina@mail.ru
}

Поступила в Редакцию 13 марта 2019 г.

В окончательной редакции 13 марта 2019 г.

Принята к публикации 19 марта 2019 г.

\begin{abstract}
Выполнено комплексное исследование структуры, фазового состава, особенностей топологии поверхности, магнитных и магнитокалорических свойств соединений $\mathrm{TbCo}_{2}, \mathrm{~Tb}_{0.3} \mathrm{Dy}_{0.7} \mathrm{Co}_{2}$ и гидрида с невысоким содержанием водорода $\mathrm{Tb}_{0.3} \mathrm{Dy}_{0.7} \mathrm{Co}_{2} \mathrm{H}_{0.5}$. Установлены особенности структуры на микро- и наноуровне, а также изменение фундаментальных и функциональных свойств при гидрировании. Показано, что введение небольшого количества водорода в кристаллическую решетку соединения $\mathrm{Tb}_{0.3} \mathrm{Dy}_{0.7} \mathrm{Co}_{2}$ приводит к увеличению, как температуры Кюри, так и магнитного момента на атомах кобальта. Тип магнитного фазового перехода из парамагнитного в магнитоупорядоченное состояние меняется с первого (в соединении $\left.\mathrm{Tb}_{0.3} \mathrm{Dy}_{0.7} \mathrm{Co}_{2}\right)$ на второй $\left(\right.$ в $\left.\mathrm{TbCo}_{2}, \mathrm{~Tb}_{0.3} \mathrm{Dy}_{0.7} \mathrm{Co}_{2} \mathrm{H}_{0.5}\right)$, что приводит к значительному снижению величины магнитокалорического эффекта.
\end{abstract}

Ключевые слова: интерметаллид, гидрид, структура, магнитокалорический эффект.

DOI: 10.21883/FTT.2019.07.47829.417

\section{1. Введение}

Соединения типа $\mathrm{RCo}_{2}$ со структурой фаз Лавеса интересны как с фундаментальной, так и практической точек зрения [1]. Относительно простая магнитная и кристаллическая структура делает их удобными модельными объектами для теоретических исследований и позволяет прогнозировать новые составы с набором уникальных свойств, которые наиболее ярко выражены в области магнитных фазовых переходов [2]. Материалы на основе $\mathrm{RCo}_{2}$ могут демонстрировать мультифункциональные свойства (такие как магнитострикционный, магнитокалорический и др. эффекты) и могут использоваться в различных областях науки и техники [3-7]. Нередко данные материалы эксплуатируются в водородсодержащих средах. Например, магнитный экохолодильник работает с использованием воды вместо фреона [8-10]. Известно [11-16], что водород, как и другие атомы легких элементов (N, C, O), проникая в кристаллическую решетку соединений, может значительным образом менять их магнитные свойств (тип магнитокристаллической анизотропии (МКА), температуру Кюри и др.). Соединения $\mathrm{RCo}_{2}$ способны поглощать до 4 атомов водорода на формульную единицу [11]. Изменение свойств соединений $\mathrm{RCo}_{2}$ зависит от количества поглощенного водорода [15]. Так, температура Кюри возрастает при малых концентрациях водорода и, наоборот, при больших концентрациях водорода наблюдается ее резкое падение. Водород может занимать два типа тетраэдрических меж- доузлий в структуре фазы Лавеса $\mathrm{C} 15$ : позиции $\mathrm{AB}_{3}$ (32e) и $\mathrm{A}_{2} \mathrm{~B}_{2}$ (96g) [17]. Согласно нейтронографическим данным для $\mathrm{RFe}_{2} \mathrm{H}_{x}(\mathrm{R}=\mathrm{Er}[18])$, при малых концентрациях водорода идет преимущественное заполнение междоузлий $\mathrm{A}_{2} \mathrm{~B}_{2}$, а при высоких - частично заполняются междоузлия обоих типов, что, по-видимому, и оказывает влияние на свойства соединений типа $\mathrm{RT}_{2}(\mathrm{~T}=\mathrm{Fe}$ и $\mathrm{Co})$.

Ранее мы исследовали магнитные свойства исходных составов типа $\mathrm{Tb}-\mathrm{Dy}-\mathrm{Co}[19,20]$, а также свойства гидридов с высоким содержанием водорода [21]. Было установлено, что внедрение трех атомов водорода в кристаллическую решетку соединения $\mathrm{Tb}_{0.3} \mathrm{Dy}_{0.7} \mathrm{Co}_{2}$ приводит к уменьшению температуры Кюри почти на $100 \mathrm{~K}$ и практически полному исчезновению магнитострикционного эффекта в невысоких (до $12 \mathrm{kOe}$ ) магнитных полях. Последнее характерно и для Fe-содержащих редкоземельных соединений, подвергнутых процедуре гидрирования [22].

Целью данной работы является сравнительное исследование микроструктуры, топологии поверхности и магнитных свойств трех соединений со структурой фаз Лавеса: $\mathrm{TbCo}_{2}, \mathrm{~Tb}_{0.3} \mathrm{Dy}_{0.7} \mathrm{Co}_{2}$ и гидрида с невысоким содержанием водорода $\mathrm{Tb}_{0.3} \mathrm{Dy}_{0.7} \mathrm{Co}_{2} \mathrm{H}_{0.5}$.

\section{2. Получение образцов и методика эксперимента}

Получение исходных образцов $\mathrm{TbCo}_{2}$ и $\mathrm{Tb}_{0.3} \mathrm{Dy}_{0.7} \mathrm{Co}_{2}$ достаточно подробно описано нами в работах [19-21]. 
Составы сплавов $\mathrm{TbCo}_{2}$ и $\mathrm{Tb}_{0.3} \mathrm{Dy}_{0.7} \mathrm{Co}_{2}$ в весовых и атомных процентах

\begin{tabular}{c|c|c|c|c|c|c|c|c}
\hline \multirow{2}{*}{ № } & \multirow{2}{*}{ Образец } & \multicolumn{4}{|c|}{ Весовые \% } & \multicolumn{3}{|c}{ Атомные \% } \\
\cline { 3 - 9 } & & $\mathrm{Co}$ & $\mathrm{Tb}$ & $\mathrm{Dy}$ & Сумма & Co & Tb & Dy \\
\hline 1 & $\mathrm{~Tb}-\mathrm{Co}$ & $43.5 \pm 1.8$ & $56.5 \pm 2.6$ & - & 100.0 & 67.5 & 32.5 & - \\
2 & $\mathrm{~Tb}-\mathrm{Dy}-\mathrm{Co}$ & $41.4 \pm 1.8$ & $18.4 \pm 2.2$ & $40.2 \pm 2.6$ & 100.0 & 65.9 & 10.9 & 23.2
\end{tabular}

Гидрирование изучаемых материалов было проведено прямой реакцией исходных образцов с газом $\mathrm{H}_{2}$ высокой чистоты с содержанием примесей $10^{-3}-10^{-4}$ масс.\%, полученным при разложении дигидрида титана $\mathrm{TiH}_{2}$. Перед процедурой гидрирования поверхность образцов активировали нагреванием в вакууме до температуры $350^{\circ} \mathrm{C}$ в течение одного часа. В процессе гидрирования использовалось невысокое давление и подача водорода порциями, чтобы избежать декомпозиции образца. Затем температуру в системе повышали для получения гидридов. Температура нагрева, длительность нагрева и длительность процесса охлаждения до комнатной температуры были подобраны опытным путем для получения однофазных образцов. Количество абсорбированного водорода определялось разницей давления газа до и после реакции. Точность такого метода определения концентрации водорода составляла \pm 0.02 атома на формульную единицу. Нами был получен однофазный образец с невысоким содержанием водорода, а именно $\mathrm{Tb}_{0.3} \mathrm{Dy}_{0.7} \mathrm{Co}_{2} \mathrm{H}_{0.5}$. Подбор оптимальных параметров гидрирования позволил избежать разрушения образца и получить его в крупнокристаллическом состоянии (а не в порошковом), что важно для выполнения сравнительных исследований с исходным образцом. Фазовый состав синтезированного соединения контролировался методом рентгеноструктурного анализа на дифрактометре ДРОН-3М.

В данной работе для исследования микроструктуры и топологии поверхности вышеприведенных соединений были использованы методы сканирующей электронной микроскопии (СЭМ) и атомно-силовой микроскопии (АСМ). На сканирующем электронном микроскопе VegaII Tescan XMU (Чехия), оснащенном детекторами отраженных и вторичных электронов и энергодисперсионным рентгеновским детектором INCA-sight (Oxford Instrement, UK), были получены снимки микроструктуры поверхности свежеприготовленных сколов образцов. Фотографии поверхности сколов образца были получены как в отраженных, так и во вторичных электронах, при ускоряющем напряжении $20 \mathrm{kV}$, токе поглощенных электронов на Со $0.1 \mathrm{nA}$. Размер зонда составил $100 \mathrm{~nm}$. Поскольку снимки, сделанные в отраженных электронах, являются наиболее информативными (дают сведения о наличии/отсутствии посторонних фаз, позволяют исследовать зерна и границы зерен [23]), ниже будут приведены именно эти фотографии.

Состав сплавов анализировался с помощью специального программного обеспечения при проведении рент- геноспектрального анализа INCA. Анализ выполняли в точке при ускоряющем напряжении $20 \mathrm{kV}$, токе поглощающих электронов на Со $0.2 \mathrm{nA}$, рабочем расстоянии $25 \mathrm{~nm}$. Размер зонда составлял $206 \mathrm{~nm}$, время набора спектра в одной точке - $70 \mathrm{~s}$. Анализ проводился по характеристическим линиям рентгеновского спектра для $\mathrm{Co}-\mathrm{CoKa}_{1,2}, \mathrm{~Tb}-\mathrm{TbLa}_{1,2}, \mathrm{Dy}-\mathrm{DyLa}_{1,2}$. В качестве стандартов использовали Со металлический, синтетические фосфаты тербия и диспрозия $\left(\mathrm{TbPO}_{4}\right.$ и $\left.\mathrm{DyPO}_{4}\right)$.

АСМ-снимки были получены на сканирующем зондовом микроскопе (С3M) SMENA-A, платформа „Solver“ компании NT-MDT (г. Зеленоград). Сканирование проводилось стандартными кремниевыми кантилеверами HA_NC ETALON c резонансными частотами $F=137-235 \mathrm{kHz}$ в полуконтактном режиме при комнатной температуре [24].

C помощью стандартного оборудования СКВИДмагнетометра получены температурные и полевые зависимости намагниченности $\mathrm{TbCo}_{2}, \mathrm{~Tb}_{0.3} \mathrm{Dy}_{0.7} \mathrm{Co}_{2}$ и гидрида $\mathrm{Tb}_{0.3} \mathrm{Dy}_{0.7} \mathrm{Co}_{2} \mathrm{H}_{0.5}$. Магнитокалорический эффект исследовался прямым методом в широком интервале температур $(80-350 \mathrm{~K})$ в полях до $12 \mathrm{kOe}$. В настоящей работе с использованием импульсных магнитных полей (до $600 \mathrm{kOe}$ ) были изучены магнитные свойства гидрида $\mathrm{Tb}_{0.3} \mathrm{Dy}_{0.7} \mathrm{Co}_{2} \mathrm{H}_{0.5}$.

\section{3. Результаты и обсуждение}

Рентгенофазовый анализ (РФА) показал, что все полученные соединения являются практически однофазными и имеют кубическую структуру типа $\mathrm{MgCu}_{2}$. Параметры решетки для исходных образцов $\mathrm{TbCo}_{2}$ и $\mathrm{Tb}_{0.3} \mathrm{Dy}_{0.7} \mathrm{Co}_{2}$ хорошо согласуются с данными работ $[5,7,19]$. Для гидрированного образца $\mathrm{Tb}_{0.3} \mathrm{Dy}_{0.7} \mathrm{Co}_{2} \mathrm{H}_{0.5}$ также был рассчитан параметр решетки $a=7.203 \AA$ и объем элементарной ячейки $V=373.72 \AA^{3}$. Установлено, что изменение объема элементарной ячейки $\Delta V / V$ составляет $0.4 \%$, в то время как в гидриде с высокой концентрацией водорода $\mathrm{Tb}_{0.3} \mathrm{Dy}_{0.7} \mathrm{Co}_{2} \mathrm{H}_{3} \Delta V / V=23.5 \%$ [21].

В таблице приведены составы сплавов $\mathrm{TbCo}_{2}$ и $\mathrm{Tb}_{0.3} \mathrm{Dy}_{0.7} \mathrm{Co}_{2}$ в весовых и атомных процентах по данным рентгеноспектрального анализа INCA. Из таблицы видно, что стехиометрия заявленных составов подтверждается результатами проведенного анализа.

На рис. $1, a, b$ приведены фотографии в отраженных электронах поверхности образцов $\mathrm{TbCo}_{2}$ и $\mathrm{Tb}_{0.3} \mathrm{Dy}_{0.7} \mathrm{Co}_{2} \mathrm{H}_{0.5}$. На сколе поверхности образца $\mathrm{TbCo}_{2}$ 


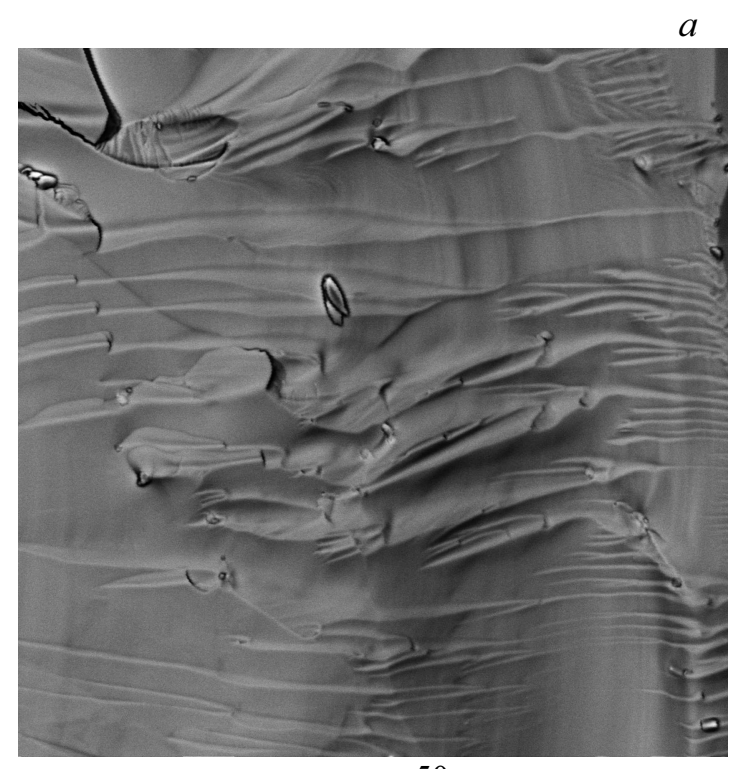

$50 \mu \mathrm{m}$

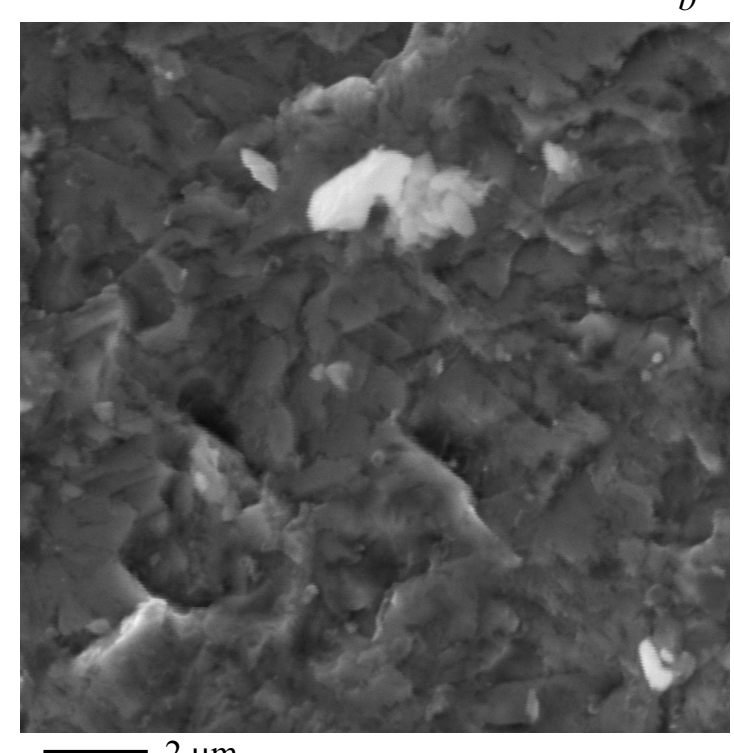

$2 \mu \mathrm{m}$

Pис. 1. Фотографии поверхности образцов $\mathrm{TbCo}_{2}(a)$ и $\mathrm{Tb}_{0.3} \mathrm{Dy}_{0.7} \mathrm{Co}_{2} \mathrm{H}_{0.5}(b)$ в отраженных электронах.

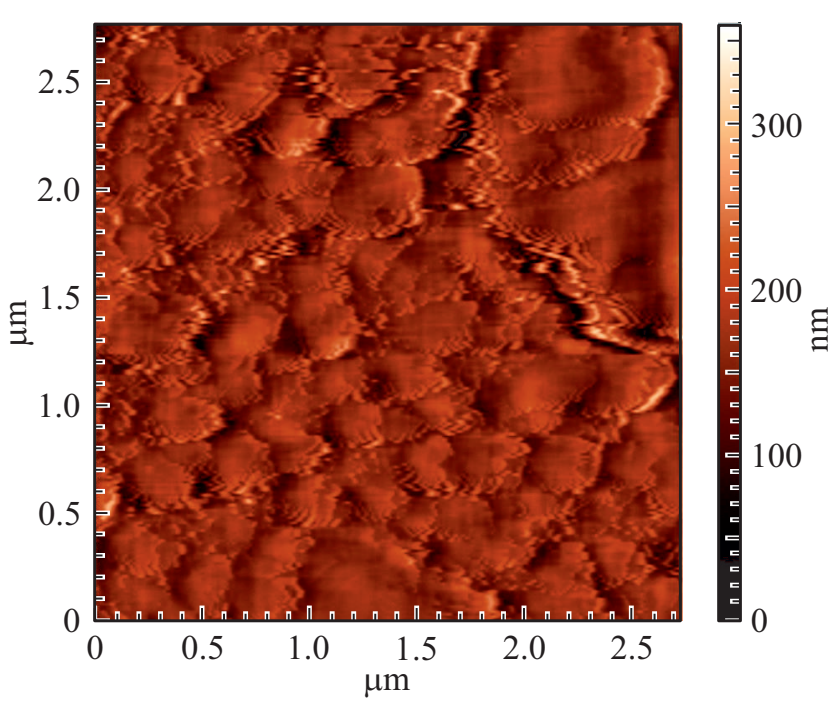

Рис. 2. АСМ-снимок поверхности гидрида $\mathrm{Tb}_{0.3} \mathrm{Dy}_{0.7} \mathrm{Co}_{2} \mathrm{H}_{0.5}$.

видна только гладкая складчатая структура скола. Похожая структура наблюдалась и для образца $\mathrm{Tb}_{0.3} \mathrm{Dy}_{0.7} \mathrm{Co}_{2}$. Следует однако отметить, что на поверхности скола $\mathrm{Tb}_{0.3} \mathrm{Dy}_{0.7} \mathrm{Co}_{2}$ были обнаружены достаточно регулярные выделения размером в несколько десятков нанометров, имеющие близкую к сферической форму, состав которых не удалось определить из-за их малых размеров. Выделения занимали не более $2-3 \%$ поверхности скола. Также было обнаружено (см. рис. $1, b$ ), что поверхность скола гидрированного образца $\mathrm{Tb}_{0.3} \mathrm{Dy}_{0.7} \mathrm{Co}_{2} \mathrm{H}_{0.5}$ очень сильно разрушена при воздействии водорода. При гидрировании происходит охрупчивание образца. На рис. 2 показан АСМ-снимок поверхности гидрида. С помо-

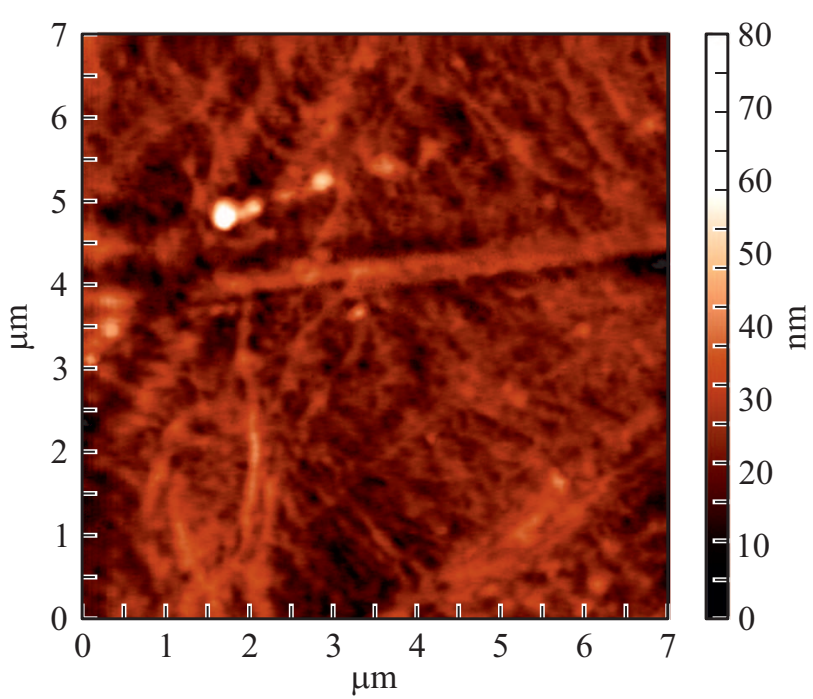

Pис. 3. АСМ-снимок поверхности образца $\mathrm{Tb}_{0.3} \mathrm{Dy}_{0.7} \mathrm{Co}_{2}$.

щью применения данного метода становится видно, что исследуемая поверхность имеет чешуйчатую структуру. Средний размер чешуек составляет приблизительно $250 \mu \mathrm{m}$. Чешуйки, в основном, имеют округлую форму с неровными краями.

АСМ-исследование было проведено и для исходных образцов $\mathrm{TbCo}_{2}$ и $\mathrm{Tb}_{0.3} \mathrm{Dy}_{0.7} \mathrm{Co}_{2}$, вырезанных из центральной области слитков. Исследование топологии поверхности обоих образцов выявило сходную структуру. На рис. 3 показан АСМ-снимок образца $\mathrm{Tb}_{0.3} \mathrm{Dy}_{0.7} \mathrm{Co}_{2}$. Бинарное соединение $\mathrm{TbCo}_{2}$ (изображение не приведено) имеет большое количество структурных элементов округлой формы (с размерами от 50 до $150 \mathrm{~nm}$ ), которые 


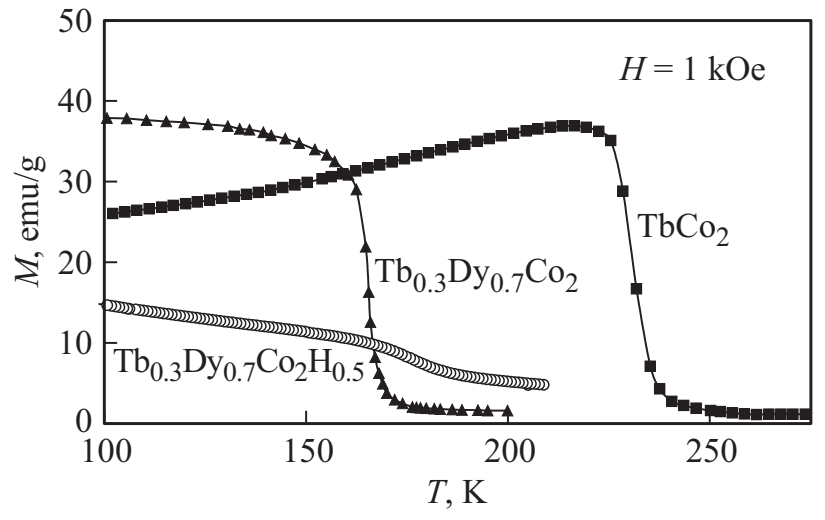

Рис. 4. Температурная зависимость намагниченности в магнитном поле $H=1 \mathrm{kOe}$ соединений $\mathrm{TbCo}_{2}, \mathrm{~Tb}_{0.3} \mathrm{Dy}_{0.7} \mathrm{Co}_{2}$ и гидрида $\mathrm{Tb}_{0.3} \mathrm{Dy}_{0.7} \mathrm{Co}_{2} \mathrm{H}_{0.5}$.

выстраиваются в виде отдельных направленных нитей. В соединении $\mathrm{Tb}_{0.3} \mathrm{Dy}_{0.7} \mathrm{Co}_{2}$ структура сплава становится более сложной. Нити диаметром порядка $100 \mathrm{~nm}$ переплетены (см. рис. 3), структура напоминает „нейронную сеть“, чем и отличается от той, что наблюдалась в бинарном соединении $\mathrm{TbCo}_{2}$.

Исследовав особенности структурного состояния трех образцов $\mathrm{TbCo}_{2}, \quad \mathrm{~Tb}_{0.3} \mathrm{Dy}_{0.7} \mathrm{Co}_{2}$ и гидрида $\mathrm{Tb}_{0.3} \mathrm{Dy}_{0.7} \mathrm{Co}_{2} \mathrm{H}_{0.5}$ на микро- и наноуровне можно заключить, что именно гидрирование вызывает наиболее значимые изменения, вследствие чего можно ожидать и значительное изменение физических и функциональных свойств материалов, подвергнутых процедуре наводораживания.

На рис. 4 представлены результаты термомагнитного анализа, проведенного в магнитном поле $H=1 \mathrm{kOe}$, для трех образцов: $\mathrm{TbCo}_{2}, \mathrm{~Tb}_{0.3} \mathrm{Dy}_{0.7} \mathrm{Co}_{2}$ и гидрида $\mathrm{Tb}_{0.3} \mathrm{Dy}_{0.7} \mathrm{Co}_{2} \mathrm{H}_{0.5}$. Видно, что характер кривых отличается. В исходных соединениях $\mathrm{TbCo}_{2}$ и $\mathrm{Tb}_{0.3} \mathrm{Dy}_{0.7} \mathrm{Co}_{2}$ наблюдается достаточно резкое падение намагниченности в области перехода из магнитоупорядоченного в парамагнитное состояние. Следует отметить, что в $\mathrm{TbCo}_{2}$ данный переход является переходом второго рода, в то время, как в частично замещенном соединении $\mathrm{Tb}_{0.3} \mathrm{Dy}_{0.7} \mathrm{Co}_{2}$ переход меняет тип с первого на второй. Самая высокая температура Кюри, определенная по производной $d M / d T$, наблюдается для $\mathrm{TbCo}_{2}$ и составляет $231 \mathrm{~K}$. В соединении $\mathrm{Tb}_{0.3} \mathrm{Dy}_{0.7} \mathrm{Co}_{2}$ температура Кюри уменьшается до значения $165 \mathrm{~K}$. Гидрирование последнего приводит к тому, что изменение намагниченности в районе $T_{C}$ становится плавным. Область перехода из магнитоупорядоченного в парамагнитное состояние в гидриде $\mathrm{Tb}_{0.3} \mathrm{Dy}_{0.7} \mathrm{Co}_{2} \mathrm{H}_{0.5}$ становится достаточно широкой, что свидетельствует о том, что переход вновь меняет тип с первого на второй. В отличие от соединения $\mathrm{TbCo}_{2}$, в гидриде $\mathrm{Tb}_{0.3} \mathrm{Dy}_{0.7} \mathrm{Co}_{2} \mathrm{H}_{0.5}$ точное определение температуры Кюри затруднено. По нашим оценкам значение температуры Кюри может находиться в интервале температур $168-170 \mathrm{~K}$, т.е. наблюдается небольшое увеличение $T_{C}$, что согласуется с данными работы [15].

Введение водорода с точки зрения расширения решетки равносильно приложению отрицательного гидростатического давления. Ожидаемое уменьшение температуры Кюри в исследованных нами гидридах с разным содержанием водорода $\mathrm{Tb}_{0.3} \mathrm{Dy}_{0.7} \mathrm{Co}_{2} \mathrm{H}_{0.5}$ и $\mathrm{Tb}_{0.3} \mathrm{Dy}_{0.7} \mathrm{Co}_{2} \mathrm{H}_{3}[21]$ вследствие возрастания объема элементарной ячейки определенное на основе литературных данных по влиянию давления на температуру Кюри $\left(d T_{C} / d P=-9 \mathrm{~K} / \mathrm{GPa}\right.$ для $\left.\mathrm{TbCo}_{2}\right)[25]$ и сжимаемость $\kappa \approx 10^{-2} \mathrm{GPa}^{-1}[26]$, показаны на рис. 5 штриховой линией. Скорость уменьшения $T_{C}$ с возрастанием объема элементарной ячейки вычисляется по формуле

$$
d \ln T_{C} / d p=-\left(\kappa / T_{C}\right) d T_{C} / d \ln V
$$

здесь $\kappa=-(d V / V) / p$. Отсюда $d T_{C} / d \ln V=d T_{C} /(d V / V)$ $=9 \mathrm{~K}$ на $1 \%$ изменения объема элементарной ячейки. Из рис. 5 видно, что экспериментально определенное уменьшение $T_{C}$ для двух систем $\mathrm{Tb}_{0.3} \mathrm{Dy}_{0.7} \mathrm{Co}_{2} \mathrm{H}_{x}$ и $\mathrm{DyCo}_{2} \mathrm{H}_{x}[15]$ меньше, чем ожидаемое в результате увеличения объема элементарной ячейки. Поэтому можно сделать заключение, что наряду с эффектом объемного расширения, действуют и другие факторы, важнейшим из которых является изменение электронной структуры соединения в результате внедрения атомов водорода в кристаллическую решетку [27,28].

Следующим шагом было исследование гидрида с малым содержанием водорода $\mathrm{Tb}_{0.3} \mathrm{Dy}_{0.7} \mathrm{Co}_{2} \mathrm{H}_{0.5}$ в области низких температур в сильных магнитных полях до $600 \mathrm{kOe} \mathrm{с} \mathrm{целью} \mathrm{выяснения} \mathrm{поведения} \mathrm{магнитных}$ моментов отдельных подрешеток (кобальта и редкоземельной подрешетки). Как видно из кривой намагничивания $M(H)$ (см. рис. 6), она демонстрирует некоторую

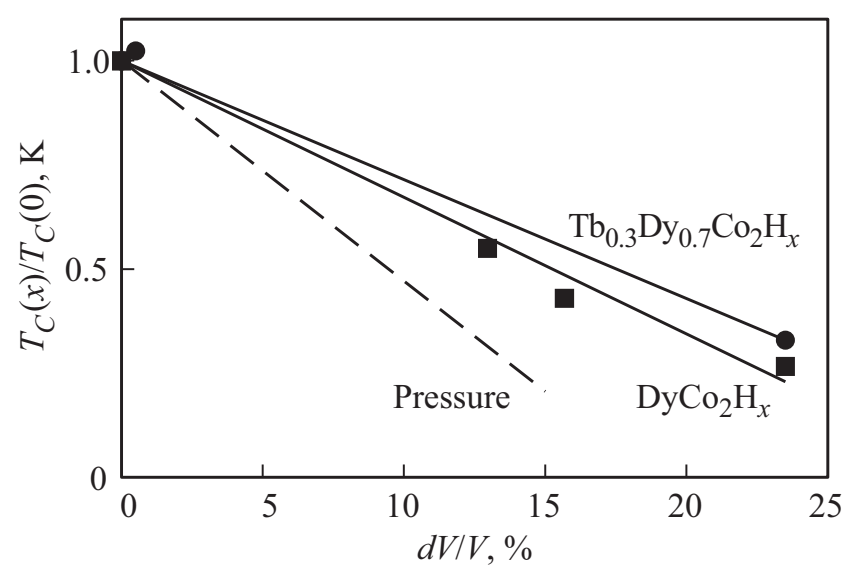

Рис. 5. Зависимость температуры Кюри для систем $\mathrm{Tb}_{0.3} \mathrm{Dy}_{0.7} \mathrm{Co}_{2} \mathrm{H}_{x}$ и $\mathrm{DyCo}_{2} \mathrm{H}_{x}[15]$ от относительного увеличения объема элементарной ячейки $\Delta V / V$ (сплошные линии) и ожидаемое изменение $T_{C}$ (пунктирная линия), определенное на основе литературных данных по влиянию гидростатического давления на температуру Кюри $[25,26]$. 


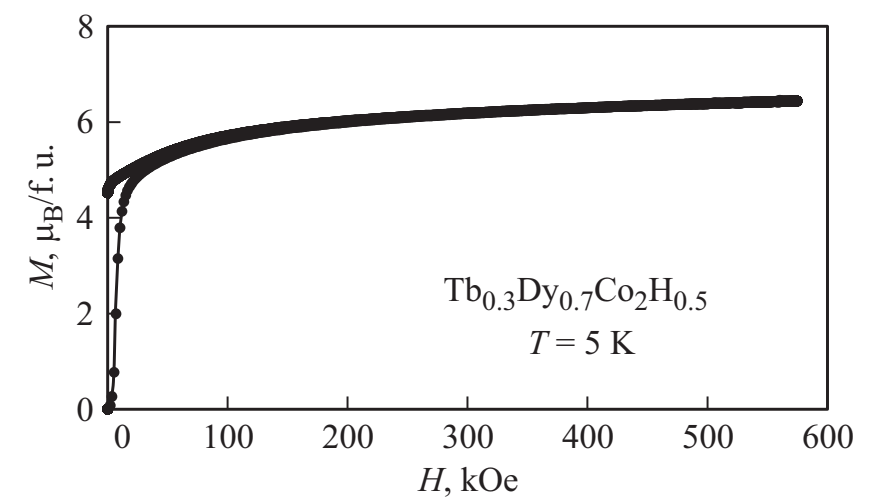

Рис. 6. Кривые намагничивания в сильных магнитных полях при $5 \mathrm{~K}$ гидрида $\mathrm{Tb}_{0.3} \mathrm{Dy}_{0.7} \mathrm{Co}_{2} \mathrm{H}_{0.5}$.

тенденцию к насыщению, причем только в очень сильных магнитных полях. Предполагается, что в данных системах может возникать неколлинеарная структура в редкоземельной подрешетке, как результат локальных флуктуаций кристаллических полей $[15,29,30]$. Наблюдаемый непрерывный рост намагниченности $M(H)$, возможно, обусловлен именно этим фактом. Разворот одной подрешетки относительно другой (подрешетки кобальта относительно редкоземельной подрешетки), не проявляется даже в полях $600 \mathrm{kOe}$, что свидетельствует о достаточно большой величине межподрешеточного обменного взаимодействия. Намагниченность в гидриде $\mathrm{Tb}_{0.3} \mathrm{Dy}_{0.7} \mathrm{Co}_{2} \mathrm{H}_{0.5}$ меньше чем в исходном соединении, что может свидетельствовать о возрастании магнитного момента на атомах Со в результате гидрирования при невысоком содержании водорода. Этот вывод полностью согласуется с данными работы [15]. Таким образом, в гидриде $\mathrm{Tb}_{0.3} \mathrm{Dy}_{0.7} \mathrm{Co}_{2} \mathrm{H}_{x}$ с $x=0.5$ at.H/f.u. происходит увеличение как температуры Кюри, так и магнитного момента на атомах кобальта.

На рис. 6 можно наблюдать гистерезисное поведение намагниченности образца $\mathrm{Tb}_{0.3} \mathrm{Dy}_{0.7} \mathrm{Co}_{2} \mathrm{H}_{0.5}$. Действительно, в нулевом поле имеется остаточная намагниченность. Ранее [19] нами были измерены петли магнитного гистерезиса для исходного состава $\mathrm{Tb}_{0.3} \mathrm{Dy}_{0.7} \mathrm{Co}_{2}$ в широкой области температур. Мы наблюдали слабый магнитный гистерезис, в том числе и при $T=4.2 \mathrm{~K}$. Отсутствие заметного по величине гистерезиса можно объяснить тем, что полная константа магнитокристаллической анизотропии $K_{1}$ невелика из-за частичной компенсации анизотропии в редкоземельной подрешетке (известно [3], что входящие в соединение $\mathrm{Tb}_{0.3} \mathrm{Dy}_{0.7} \mathrm{Co}_{2}$ редкоземельные ионы тербия и диспрозия имеют различные знаки одноионных констант МКА). Вопрос об упорядочении атомов водорода в системах $\mathrm{RCo}_{2}-\mathrm{H}$ при малых концентрациях водорода и его влияние на МКА требует дополнительных исследований, поскольку в системах $\mathrm{R}-\mathrm{H}$ такое упорядочение атомов водорода наблюдалось экспериментально [31,32].
Как уже отмечалось выше, основными функциональными свойствами соединений $\mathrm{RCo}_{2}$ являются гигантская магнитострикция и магнитокалорический эффект, которые проявляются в области температуры Кюри $[5-7,33,34]$. Для исходных соединений $\mathrm{Tb}_{0.3} \mathrm{Dy}_{0.7} \mathrm{Co}_{2}$ и $\mathrm{TbCo}_{2}$, демонстрирующих переход первого и второго рода, соответственно, эти функциональные свойства подробно исследованы в работе [5]. Магнитокалорический эффект в $\mathrm{Tb}_{0.3} \mathrm{Dy}_{0.7} \mathrm{Co}_{2}$ в два раза больше, чем в $\mathrm{TbCo}_{2}$. Напомним, что температуры Кюри равны 165 и $231 \mathrm{~K}$ для $\mathrm{Tb}_{0.3} \mathrm{Dy}_{0.7} \mathrm{Co}_{2}$ и $\mathrm{TbCo}_{2}$ соответственно. Было также установлено [5], что в области $T_{C}$ между двумя эффектами (магнитокалорическим и магнитострикционным) наблюдается полная корреляция, поскольку оба эффекта одинаково зависят от величины намагниченности $[3,35,36]$. В данной работе мы приводим исследование магнитокалорического эффекта для гидрида $\mathrm{Tb}_{0.3} \mathrm{Dy}_{0.7} \mathrm{Co}_{2} \mathrm{H}_{0.5}$, поскольку исследование магнитострикции затруднено из-за повышенной хрупкости образцов.

На рис. 7 показана температурная зависимость магнитокалорического эффекта соединения $\mathrm{Tb}_{0.3} \mathrm{Dy}_{0.7} \mathrm{Co}_{2}$ и его гидрида, измеренная прямым методом. Видно, что величина МКЭ уменьшается приблизительно в восемь раз в результате гидрирования. Основной причиной уменьшения МКЭ при гидрировании может быть тот факт, что тип магнитного фазового перехода изменился с первого на второй. В работах [37,38] на примере большого количества замещенных составов типа $\left(\mathrm{RR}^{\prime}\right)(\mathrm{Co}, \mathrm{T})_{2}$ было показано, что основными факторами, влияющими на величину МКЭ являются величина температуры Кюри и тип перехода. Выявлены два обязательных условия: величина МКЭ может быть значительной только в области низких температур, если $T_{C}<200 \mathrm{~K}$ и если переход из парамагнитного в магнитоупорядоченное состояние является переходом первого рода. Соединение

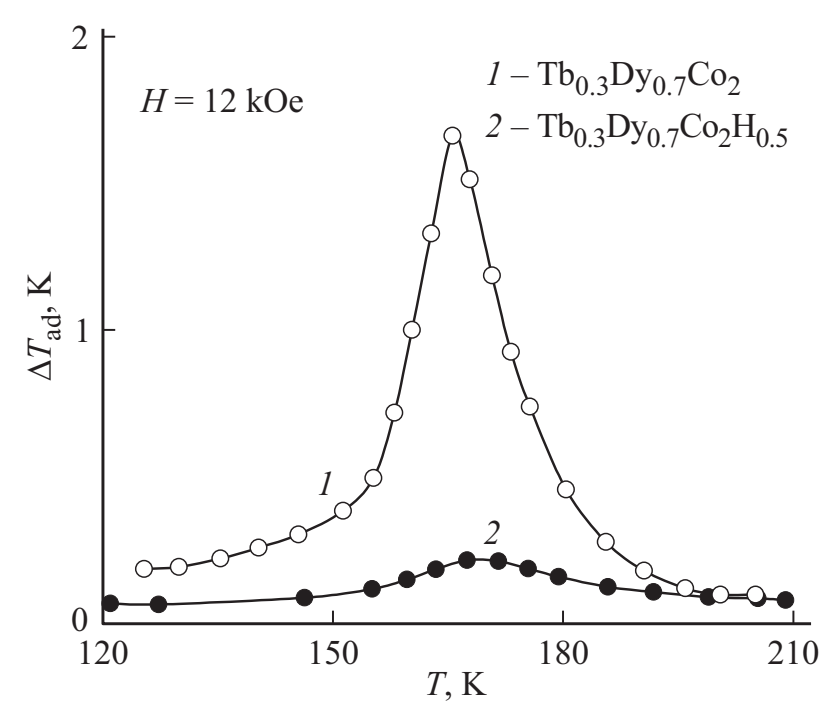

Рис. 7. Температурная зависимость магнитокалорического эффекта для соединения $\mathrm{Tb}_{0.3} \mathrm{Dy}_{0.7} \mathrm{Co}_{2}$ и его гидрида. 
$\mathrm{Tb}_{0.3} \mathrm{Dy}_{0.7} \mathrm{Co}_{2}$ удовлетворяет обоим условиям. Если в результате различных замещений как в подрешетке редкоземельного металла, так и в подрешетке кобальта (на атомы кремния, алюминия и др.), одно из условий нарушилось, например, произошла смена типа перехода с первого на второй, а температура Кюри, по-прежнему, не превышает $200 \mathrm{~K}$, то в таком многокомпонентном соединении типа $\left(\mathrm{RR}^{\prime}\right)(\mathrm{Co}, \mathrm{T})_{2}$ величина МКЭ уменьшится значительно.

Данное исследование показывает, что не только атомы замещения, но и атомы внедрения в соединениях $\mathrm{RCo}_{2}$ способны изменить (в нашем случае, повысить) температуру перехода из парамагнитного в магнитоупорядоченное состояние $\left(T_{C}\right.$, однако, остается меньше $200 \mathrm{~K}$ для $\left.\mathrm{Tb}_{0.3} \mathrm{Dy}_{0.7} \mathrm{Co}_{2} \mathrm{H}_{0.5}\right)$, изменить тип перехода (с первого на второй), сохранив при этом обнаруженную ранее для замещенных составов $\left(\mathrm{RR}^{\prime}\right)(\mathrm{Co}, \mathrm{T})_{2}$ тенденцию значительного снижения величины МКЭ.

\section{4. Заключение}

Проведено комплексное исследование структурных особенностей и функциональных свойств соединений типа $\mathrm{Tb}-\mathrm{Dy}-\mathrm{Co}-\mathrm{H}$ со структурой фаз Лавеса. Для исследования микроструктуры соединений $\mathrm{TbCo}_{2}$, $\mathrm{Tb}_{0.3} \mathrm{Dy}_{0.7} \mathrm{Co}_{2}$ и гидрида с невысоким содержанием водорода $\mathrm{Tb}_{0.3} \mathrm{Dy}_{0.7} \mathrm{Co}_{2} \mathrm{H}_{0.5}$ были использованы два метода: метод сканирующей электронной микроскопии и атомно-силой микроскопии. Использование метода АСМ позволило увидеть тонкую наноструктуру образцов. Введение водорода в кристаллическую решетку $\mathrm{Tb}-\mathrm{Dy}-\mathrm{Co}$ вызвало охрупчивание образцов. Установлено, что гидрирование образцов до невысокой концентрации водорода $x=0.5$ at. H/f. u. приводит к увеличению температуры Кюри и увеличению магнитного момента на атомах Со. Однако, магнитокалорический эффект уменьшается значительно, в результате того, что тип фазового перехода в соединении $\mathrm{Tb}_{0.3} \mathrm{Dy}_{0.7} \mathrm{Co}_{2}$ меняется с первого на второй. Учет полученных выше результатов важен при конструировании рабочих тел магнитных рефрижераторов, а также сенсоров и датчиков различного назначения из материалов на основе соединений типа $\mathrm{RCo}_{2}$.

\section{Благодарности}

Авторы выражают благодарность Г. Друлису за получение гидрированного образца и Д. Горбунову за оказанную помощь при проведении измерений в сильных магнитных полях.

\section{Финансирование}

Исследование выполнено за счет гранта Российского научного фонда (проект № 18-13-00135).

\section{Конфликт интересов}

Авторы заявляют, что у них нет конфликта интересов.

\section{Список литературы}

[1] N.H. Duc, T. Goto. Itinerant electron metamagnetism of Co sublattice in the lanthanide-cobalt intermetallics. in Handbook on Physics and Chemistry of Rare Earths / Ed. K.A. Gschneidner, Jr. LeRoy Eyring. Elsevier Science, Amsterdam. (1999). V. 26. 177 p.

[2] E. Gratz, A.S. Markosyan. J. Phys. Condens. Matter 13, R385 (2001).

[3] К.П. Белов. Магнитострикционные явления и их технические приложения. Наука, М. (1987). 160 с.

[4] A.M. Tishin, Y.I. Spichkin. The Magnetocaloric Effect and Its Applications. Institute of Physics, Bristol (2003). 475 p.

[5] I.S. Tereshina, J. Ćwik, E.A. Tereshina, G. Politova, G. Burkhanov, V. Chzhan, A.S. Ilyushin, M. Miller, A. Zaleski, K. Nenkov, L. Schultz. IEEE Trans. Mag. 50, 2504604 (2014).

[6] V.B. Chzhan, I.S. Tereshina, A.Yu. Karpenkov, E.A. TereshinaChitrova. Acta Mater. 154, 303 (2018).

[7] I. Tereshina, G. Politova, E. Tereshina, G. Burkhanov, O. Chistyakov, S. Nikitin. J. Phys.: Conf. Ser. 266, 012077 (2011).

[8] E. Bruck. J. Physics D 38, R381 (2005).

[9] V. Franco, J.S. Blazquez, J.J. Ipus, J.Y. Law, L.M. MorenoRamirez, A. Conde. Prog. Mater. Sci. 93, 112 (2018).

[10] A. Kitanovski, J. Turek, U. Tomc, U. Plaznik, M. Ožbolt, A. Poredoš. Magnetocaloric Energy Conversion From Theory to Applications. Springer International Publishing Switzerland, Cham (2015). 456 p.

[11] G. Wiesenger, G. Hilscher. Magnetism of hydrides. in Handbook of Magnetic Materials / Ed. K.H.J. Buschow. Elsevier, Amsterdam (2008). V. 17. 293 p.

[12] S. Nikitin, I. Tereshina, E. Tereshina, W. Suski, H. Drulis. J. Alloys Comp. 451, 477 (2008).

[13] H. Fujii, H. Sun. Interstitially modified intermetallics of rareearth-elements. in Handbook of Magnetic Materials / Ed. K.H.J. Buschow. Elsevier, Amsterdam (1995). V. 9, 304 p.

[14] E.A. Tereshina, H. Drulis, Y. Skourski, I. Tereshina. Phys. Rev. B 87, 214425 (2013).

[15] Н.В. Мушников, В.С. Гавико, Т. Гото. ФММ 100, 24 (2005).

[16] S.A. Nikitin, I.S. Tereshina, N.Yu. Pankratov, Yu.V. Skourski. Phys. Rev. B 63, 134420 (2001).

[17] D.P. Shoemaker, C.B. Shoemaker. J. Less-Common Met. 68, 43 (1979).

[18] T. De Saxce, Y. Berthier, D. Fruchart. J. Less-Common Met. 107, 35 (1985).

[19] Г.А. Политова, И.С. Терёшина, Г.С. Бурханов, О.Д. Чистяков, В.Б. Чжан, Т.П. Каминская, В.В. Попов, А. Залески, В.И. Нижанковский. ФТТ 53, 1925 (2011).

[20] Г.С. Бурханов, И.С. Терёшина, Г.А. Политова, О.Д. Чистяков, Г. Друлис, А. Залески. Докл. РАН 440, 611 (2011).

[21] Г.А. Политова, И.С. Терешина, С.А. Никитин, Ю.Б. Патрикеев, Т.Г. Соченкова, В.Н. Вербецкий, А.А. Саламова, М.В. Макарова. ФТТ 47, 1834 (2005).

[22] S.A. Nikitin, I.S. Tereshina, V.N. Verbetsky, A.A. Salamova, K.P. Skokov, N.Yu. Pankratov, Yu.V. Skourski, N.V. Tristan, V.V. Zubenko, I.V. Telegina. J. Alloys Comp. 322, 42 (2001). 
[23] Растровая электронная микроскопия для нанотехнологий. Методы и применение / Под ред. У. Жу, Ж.Л. Уанга. Пер. с англ. под ред. Т.П. Каминской. БИНОМ. Лаборатория знаний, М. (2013). 582 с.

[24] И.В. Яминский. Основы атомно-силовой микроскопии / Под ред. И.В. Яминский, Д.В. Багров. НОУДПО Институт информационных технологий „АйТи“, М. (2011). 128 с.

[25] E. Burzo, P. Vlaic, D.P. Kozlenko, S.E. Kichanov, N.T. Dang, E.V. Lukin, B.N. Savenko. J. Alloys Comp. 551, 702 (2013).

[26] M. Brouha, K.H.J. Buschow. J. Phys. F 3, 2218 (1973).

[27] E.A. Tereshina, S. Khmelevskyi, G. Politova, T. Kaminskaya, H. Drulis, I.S. Tereshina. Sci. Rep. 6, 22553 (2016).

[28] E.A. Tereshina, H. Yoshida, A.V. Andreev, I.S. Tereshina, K. Koyama, T. Kanomata. J. Phys. Soc. Jpn 76, Suppl. A, 82 (2007).

[29] А.С. Маркосян. ФТТ 23, 1656 (1981).

[30] N.V. Mushnikov, N.K. Zajkov, M.I. Bartashevich, T. Goto, H. Aruga-Katori, M. Yamaguchi, I. Yamamoto. J. Magn. Magn. Mater. 167, 229 (1997).

[31] P. Vajda. In: Handbook on the Physics and Chemistry of Rare Earths / Ed. K.A. Gschneidner, Jr., LeRoy Eyring. North-Holland, Amsterdam (1995). V. 20, 207 p.

[32] G.S. Burkhanov, N.B. Kolchugina, E.A. Tereshina, I.S. Tereshina, G.A. Politova, V.B. Chzhan, D. Badurski, O.D. Chistyakov, M. Paukov, H. Drulis, L. Havela. Appl. Phys. Lett. 104, 242402 (2014).

[33] I. Tereshina, G. Politova, E. Tereshina, S. Nikitin, G. Burkhanov, O. Chistyakov, A. Karpenkov. J. Phys.: Conf. Ser. 200, 092012 (2010).

[34] I. Tereshina, G. Politova, E. Tereshina, J. Cwik, S. Nikitin, O. Chistyakov, A. Karpenkov, D. Karpenkov, T. Palewski. J. Phys.: Conf. Ser. 303, 012024 (2011).

[35] Л.Д. Ландау, Е.М. Лифшиц. Статистическая физика. Наука, М. (1976). $584 \mathrm{c}$.

[36] A. del Moral. Handbook of Magnetostriction and Magnetostrictive Materials. Del Moral Publ., University of Zaragoza, Spain. (2008). 148 p.

[37] N.H. Duc, D.T. Kim Anh, P.E. Brommer. Physica B 319, 1 (2002).

[38] J. Prokleska, J. Vejpravova, D. Vasylyev, S. Danis, V. Sechovsky. J. Magn. Magn. Mater. 290-291, 676 (2005).

Редактор Т.Н. Василевская 